\title{
Die Angiotensinase-Aktivität der $\alpha$-L-Asparaginyl-Aminopeptidase im Serum von Normalpersonen, bei hepatobiliären Krankheiten und während der Schwangerschaft
}

\author{
Von H. Kaulfausen, P. Pfeiffer ${ }^{1}$ ) und H. Breuer \\ Aus dem Institut für Klinische Biocbemie und Klinische Chemie der Universität Bonn
}

(Eingegangen am 9. August 1971)

\begin{abstract}
Die Angiotensinase-Aktivität der $\alpha$-L-Asparaginyl-Aminopeptidase (EC 3.4.1.2) ${ }^{2}$ ) wurde mit einem optischen Test im Serum von 25 Normalpersonen (10 Frauen und 15 Männer), von 15 Schwangeren und von 14 Patienten mit hepatobiliären Krankheiten bestimmt. Die mittlere Aktivität der $\alpha$-L-Asparaginyl-Aminopeptidase, ausgedrückt in $U / 1$, betrug bei 10 normalen Frauen $7,25 \pm 1,36$, bei 15 normalen Männern 7,38 $\pm 2,00$, bei 15 Schwangeren 7,40 $\pm 3,07$ sowie bei 6 Patienten mit Verschlußikterus und/oder Gallengangsstrikturen 42,10, bei 4 Patienten mit Cholelithiasis 25,25 und bei 4 Patienten mit Leberparenchymerkrankungen 13,93. Zwischen den Aktivitäten der $\alpha$-L-Asparaginyl-Aminopeptidase und der alkalischen Phosphatase bestand bei den Patienten mit hepatobiliären Krankheiten einc positive Korrelation $(+0,713)$, die statistisch signifikant war $(0,005>p>0,001)$. Die vorliegenden Ergebnisse legen die Vermutung nahe, daß die herabgesetzte vasopressorische Reaktion auf intravenöse Infusionen von $\alpha$-L-Asparaginyl-Angiotensin II bei Patienten mit dekompensierter Lebercirrhose durch die erhöhte Aktivität der $\alpha$-L-Asparaginyl-Aminopeptidase bedingt ist.
\end{abstract}

\section{The angiotensinase activity of $\alpha$-L-asparaginyl aminopeptidase in the serum of normal subjects, in patients with bepatobiliary diseases and during pregnancy}

The angiotensinase activity of $\alpha$-L-asparaginyl aminopeptidase (EC 3.4.1.2) was measured by means of an optical test in the serum of 25 normal subjects (10 females and 15 males), 15 pregnant women and 14 patients with hepatobiliary diseases. The mean values for the activity of $\alpha$-L-asparaginyl aminopeptidase, expressed as $U / 1$, were $7.25 \pm 1.36$ in 10 normal females, $7.38 \pm 2.00$ in 15 normal males, $7.40 \pm 3.07$ in 15 pregnant women, and 42.10 in 6 patients with obstructive jaundice and/or strictures of the biliary tract, 25.25 in 4 patients with cholelithiasis and 13.93 in 4 patients with diseases of liver parenchyma. A positive correlation $(+0.713)$, which was statistically significant $(0.005>p>0.001)$, was found between the activities of $\alpha$-L-asparaginyl aminopeptidase and alkaline phosphatase. On the basis of the results reported here it is concluded that the diminished pressor action of intravenously administered $\alpha$-L-asparaginyl angiotensin II, observed in patients with decompensated liver cirrhosis, may be due to an increased activity of the $\alpha$-L-asparaginyl aminopeptidase.

Die stärkste pressorische Substanz im menschlichen Plasma, das Oktapeptid Angiotensin II, wird durch sogenannte Angiotensinasen inaktiviert. Bisher sind verschiedene Peptidasen mit Angiotensin-spaltender Wirkung beschrieben worden; dabei handelt es sich im einzelnen um folgende Enzyme:

a) die Endopeptidasen Pepsin, Trypsin und Chymotrypsin $(1-3)$,

b) die Carboxypeptidasen, die in der Niere des Schweines, in der Leber des Kaninchens und im Urin des Menschen vorkommen (4), allerdings noch nicht im menschlichen Plasma nachgewiesen werden konnten, sowie

c) die Aminopeptidasen.

Bei der Untersuchung der Aminopeptidasen zeigte sich, $\mathrm{da} \beta$ die $\alpha-\mathrm{L}-\mathrm{Verbindungen} \mathrm{rascher} \mathrm{gespalten} \mathrm{werden} \mathrm{als}$ diejenigen Oktapeptide, die am Aminoende $\alpha$-D-Asparagin, $\beta$-D-Asparagin oder $\beta$-L-Asparagin enthalten (5). Die $\alpha$-L-Asparaginyl-Aminopeptidase $\left(\mathrm{A}_{1} \mathrm{AP}\right)$ inaktiviert nur $\alpha$-L-Asparaginyl-Angiotensin II (pH-Optimum 7,4),

1) Teil der Dissertation P. Pfriffer, Medizinische Fakultät der Universität Bonn, 1971.

2) Enzym: $\mathrm{A}_{1} \mathrm{AP}=$ Amino-acyl-oligopeptid-Hydrolase (EC 3.4.1.2) während die $\alpha$-L-Aspartyl-Aminopeptidase $\left(\mathrm{A}_{2} \mathrm{AP}\right)$ nur $\alpha$-L-Aspartyl-Angiotensin II (pH-Optimum 6,8) spaltet $(6,7)$. KLAUS und Mitarbeiter (8) fanden, $\mathrm{da} \beta$ die Abspaltung von $\alpha$-L-Asparagin der geschwindigkeitsbestimmende Schritt bei der enzymatischen Hydrolyse von $\alpha$-L-Asparaginyl-Angiotensin II ist.

Úber die pathophysiologische Bedeutung der Angiotensinasen ist nur wenig bekannt. So wurden bei $\mathrm{Pa}-$ tienten mit renaler Hypertonie erhöhte (9) oder erniedrigte $(10,11)$, meist aber unveränderte (12-16) Angiotensinase-Aktivitäten beschrieben. Dagegen wurde im Serum von Patienten mit hepatobiliären Krankheiten stets ein Anstieg der Angiotensinase-Aktivität beobachtet (16-18). Während der Schwangerschaft (3. Trimenon) fanden sich zum Teil erhöhte $(9,12,19-22)$, zum Teil normale $(16,18)$ Werte für die AngiotensinaseAktivität.

Die in verhältnismäßig geringer Anzahl vorliegenden und mit unterschiedlicher Methodik gewonnenen Ergebnisse veranlaßten uns, die Angiotensinase-Aktivität der $\alpha$-L-Asparaginyl-Aminopeptidase während der Schwangerschaft, besonders aber bei Patienten mit hepatobiliären Krankheiten zu bestimmen. Diese Untersuchungen sollten dazu beitragen, den erniedrigten 
Blutdruck bei chronischen Leberkrankheiten sowie die verminderte vasopressorische Reaktion auf Infusionen von $\alpha$-L-Asparaginyl-Angiotensin II bei dekompensierter Lebercirrhose $(23,24)$ zu erklären.

\section{Methodik}

Reagenzien, Puffer und Pbarmaka

Alle Reagenzien waren von p. a.-Reinheitsgrad (Merck, Darmstadt). 2-Osoglutarsäure und $\mathrm{NADH}$ wurden von Boehringer Mannheim bezogen. Es wurden folgende Puffer verwendet: 0,067 M Phosphat-Puffer (pH 6,4), 0,067 M Phosphat-Puffer ( $\mathrm{pH}$ 7,2) und 0,67 Mr Phosphat-Puffer ( $\mathrm{pH} 7,2)$.

Lösungen: 1,6 $\mathrm{M}$ und 2,8 $\mathrm{M}$ Ammoniumsulfat-Lösung.

Hypertensin Ciba:Trockenampullen mit $2,5 \mathrm{mg} \alpha$-L-Asparaginyl${ }^{5}$ Valin-Angiotensin II (Ciba, Basel/Schweiz).

\section{Enoj'me}

Folgende Enzyme wurden von Boehringer Mannheim bezogen: L-Asparagin-Amidohydrolase (Asparaginase [EC 3.5.1.1]), Aspartat-Aminotransferase (EC 2.6.1.1) und L-Malat: NAD-Oxydoreduktase (EC 1.1.1.37).

\section{Normalpersonen}

Als Normalpersonen dienten 10 Frauen und 15 Männer (Alter 22-48 Jahre), die frei von erkennbaren Leberkrankheiten waren.

\section{Schwangere}

Die Seren stammten von 15 Frauen im 3. Trimenon (Alter 21 bis 39 Jahre). Zwei Schwangere hatten hepatobiliäre Komplikationen.

\section{Leberkranke}

Es handelte sich um neun weibliche (Alter 25-71 Jahre) und fünf männliche Patienten (Alter 32-67 Jahre) der Chirurgischen Universitätsklinik Bonn, bei denen die klinische Diagnose

a) biliäre Erkrankung mit Verschlußsyndrom $(\mathrm{N}=10)$,

b) Lebercirrhose $(\mathrm{N}=3)$ bzw.

c) Pankreastumor $(\mathrm{N}=1)$

gestellt worden war.

\section{Gewinnung des Serums}

Blut wurde aus einer Cubitalvene entnommen und 20-30 Min. nach der Entnahme $10 \mathrm{Min}$. bei $1500 \mathrm{~g}$ zentrifugiert. Die Inkubationsversuche wurden spätestens $3 \mathrm{Stdn}$. nach der Blutentnahme begonnen.

Bestimmung der Aktivität der alkalischen Phosphatase und der AlaninAminotransferase im Serum

Die Bestimmungen exfolgten nach den Angaben der jeweiligen Biochemica Test Combinationen, Boehringer Mannheim.

\section{Bestimmung der Aktivität der $\alpha$-L-Asparaginyl-Aminopeptidase}

Die Bestimmung erfolgte in Anlehnung an OelKers und GoldACKER (25); anstelle der aus Meerschweinchenblut gewonnenen Asparaginase wurde eine Enzympräparation aus E. coli verwendet. Jeweils $0,5 \mathrm{ml}$ der $1: 1$ mit 0,9 proz. $\mathrm{NaCl}$-Lösung verdünnten Serumproben wurden mit $5 \mathrm{mg}$ Hypertensin $\mathrm{Ciba}$, das in $1,5 \mathrm{ml}$ $0,067 \mathrm{M}$ Phosphat-Puffer ( $\mathrm{pH} 6,4$ ) gelöst war, $60 \mathrm{Min}$. bei $37^{\circ}$ im Schüttelthermostaten inkubiert. Die Substratkonzentration betrug 1,8 mM. Anschließend wurde die Inkubationslösung zur Eiweißfällung $10 \mathrm{Min}$. auf $100^{\circ}$ erhitzt, das Präzipitat $20 \mathrm{Min}$. bei $1500 \mathrm{~g}$ abzentrifugiert und verworfen. $1,5 \mathrm{ml}$ des Uberstandes

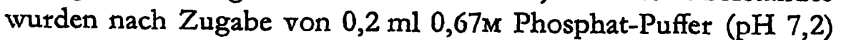
mit $0,2 \mathrm{ml}$ Asparaginase-Lösung $60 \mathrm{Min}$. bei $37^{\circ}$ inkubiert. Die entstehende Asparaginsäure wurde im optischen Test bei Raumtemperatur quantitativ bestimmt. Zu diesem $Z$ weck wurden nach-

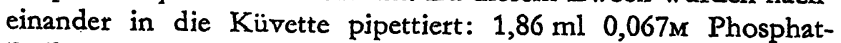
Puffer ( $\mathrm{pH} 7,2$ ), $0,02 \mathrm{ml}$ einer Aspartat-Aminotransferase-Lösung (2,5 mg Protein/ml in $1,6 \mathrm{M}$ Ammoniumsulfat), $0,02 \mathrm{ml}$ einer Malatdehydrogenase-Lösung (2,5 mg Protein/ml in $2,8 \mathrm{M} \mathrm{Am}-$ moniumsulfat), $0,05 \mathrm{ml}$ einer 1 proz. NADH-Lösung sowie $1 \mathrm{ml}$ der zu untersuchenden Lösung. Der Küvetteninbalt wurde gemischt und die Extinktion $E_{1}$ abgelesen. Durch Zugabe von $0,05 \mathrm{ml}$ 2-Oxoglutarat-Lösung wurde der optische Test gestartet. Nach $10 \mathrm{Min}$. wurde die Extinktion $\mathrm{E}_{2}$ abgelesen. Mit Hilfe der folgenden Umrechnungsformel wird die Aktivität der $\alpha$-L-Asparaginyl-Aminopeptidase $\left(A_{1} A P\right)$ errechnet:

$$
A_{1} A P-A k t i v i t a ̈ t[U / 1]=\left(E_{1}-E_{2}-E_{\text {Blindwert }}\right) \cdot 97,933
$$

$\mathrm{Da}$ die Asparaginase ebenfalls eine Angiotensinase-Aktivität entfaltet, ist von allen $\triangle E-W e r t e n$ ein entsprechender Blindwert abzuziehen; die mittlere Blindwert-Extinktion aus sechs Kontrollansätzen betrug 0,028 .

\section{Enzymeinbeit}

Als eine Enzymeinheit (U) wird diejenige Enzymmenge definiert, die unter standardisierten Bedingungen ( $\mathrm{pH} 6,4 ; 37^{\circ}$ ) $1 \mu \mathrm{Mol}$ Asparaginyl-Angiotensin II/Min. umsetzt.

\section{Ergebnisse}

\section{Untersucbungen zur Kinetik der $\alpha$-L-Asparaginyl-Amino- peptidase}

Die Abspaltung von Asparagin am Aminoende von Asparaginyl-Angiotensin II durch die $\alpha$-L-AsparaginylAminopeptidase $\left(A_{1} A P\right)$ in Abhängigkeit von der Zeit ist in Abbildung 1 dargestellt. Die Hydrolyse folgt einer Reaktion nullter Ordnung. In Abbildung 2 ist die Aktivität der $A_{1} A P$ in Abhängigkeit von der Substratkonzentration wiedergegeben. Die Messungen wurden bei $37^{\circ}$ und einem pH-Wert von 6,4 durchgeführt. Aus dem Verlauf der Kurve und der Transformation nach LinetveAver und Burk (26) geht hervor, daB die Abspaltung von Asparagin der MICHAELIS-MENTEN-Kinetik gehorcht. Der $\mathrm{K}_{\mathrm{m}}$-Wert für Asparaginyl-Angiotensin II beträgt $0,91 \mathrm{~mm}$.

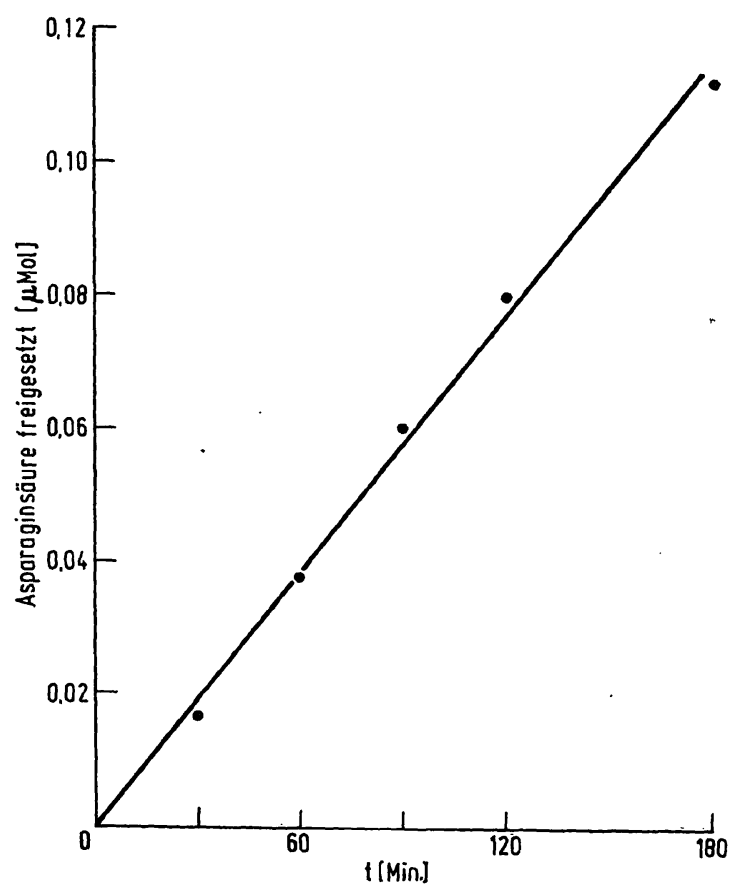

Abb. 1

Inaktivierung von Asparaginyl-Angiotensin II durch Abspaltung von Asparagin durch die $\alpha$-L-Asparaginyl-Aminopeptidase in Abhängigkeit Von der Zeit. Jeweils $1,5 \mathrm{ml}$ Serum (1:1 verdünnt) wurden mit $15 \mathrm{~m}$ Asparaginyl-Angiotensin II in $6 \mathrm{ml} 0,067 \mathrm{M}$ Phosphat-Puffer (pH 6,4) bei $37^{\circ}$ inkubiert. Die Bestimmung von Asparagin erfolgte nach Oberführung in Asparaginsäure im optischen Test; Einzelheiten vgl 
Tab. 1

Aktivitäten der a-L-Asparaginyl-Aminopeptidase (A,AP), der alkalischen Phosphatase (AP) und der Alanin-Aminotransferase (GPT) im Serum bei 14 Patienten mit hepatobiliären Krankheiten

\begin{tabular}{|c|c|c|c|c|c|}
\hline $\mathrm{Nr}$. & Geschlecht & Klinische Diagnose & $\begin{array}{l}\mathrm{A}_{1} \mathrm{AP}^{\mathrm{A}} \\
{[\mathrm{U} / 1]}\end{array}$ & $\begin{array}{l}\text { ivität der } \\
\text { AP } \\
{[U / I]}\end{array}$ & $\begin{array}{l}\text { GPT } \\
{[U / I]}\end{array}$ \\
\hline $\begin{array}{r}1 \\
2 \\
3 \\
4 \\
5 \\
6 \\
7 \\
8 \\
9 \\
10 \\
11 \\
12 \\
13 \\
14\end{array}$ & 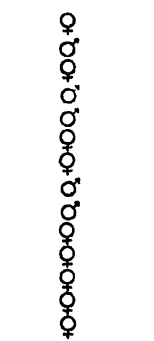 & $\begin{array}{l}\text { Gallengangsstrikturen, M. BOECK } \\
\text { Verschlußikterus bei Metastasen } \\
\text { Cholelithiasis } \\
\text { Gallengangsstrikturen, Echinokokkus der Leber } \\
\text { Verschlußikterus bei Tumor } \\
\text { Verschlußikterus bei Ca des D. hepaticus } \\
\text { Cholelithiasis } \\
\text { Cholelithiasis } \\
\text { hochgradige Lebercirrhose, portale Hypertension } \\
\text { Cholelithiasis } \\
\text { iatrogener Verschluß des Ductus hepaticus } \\
\text { Lebercirrhose } \\
\text { Pankreaskopf-Ca mit Lebermetastasen } \\
\text { hochgradige Lebercirrhose, portale Hypertension }\end{array}$ & $\begin{array}{l}82,87 \\
57,11 \\
39,43 \\
38,49 \\
30,97 \\
26,78 \\
23,73 \\
21,45 \\
16,84 \\
16,37 \\
16,37 \\
13,96 \\
13,24 \\
11,69\end{array}$ & $\begin{array}{l}750 \\
340 \\
740 \\
416 \\
400 \\
350 \\
260 \\
226 \\
190 \\
432 \\
212 \\
241 \\
154 \\
380\end{array}$ & $\begin{array}{c}90,0 \\
33,5 \\
28,0 \\
18,0 \\
\overline{22,5} \\
20,4 \\
15,7 \\
\overline{56,3} \\
45,0 \\
33,5 \\
27,0 \\
20,3\end{array}$ \\
\hline
\end{tabular}

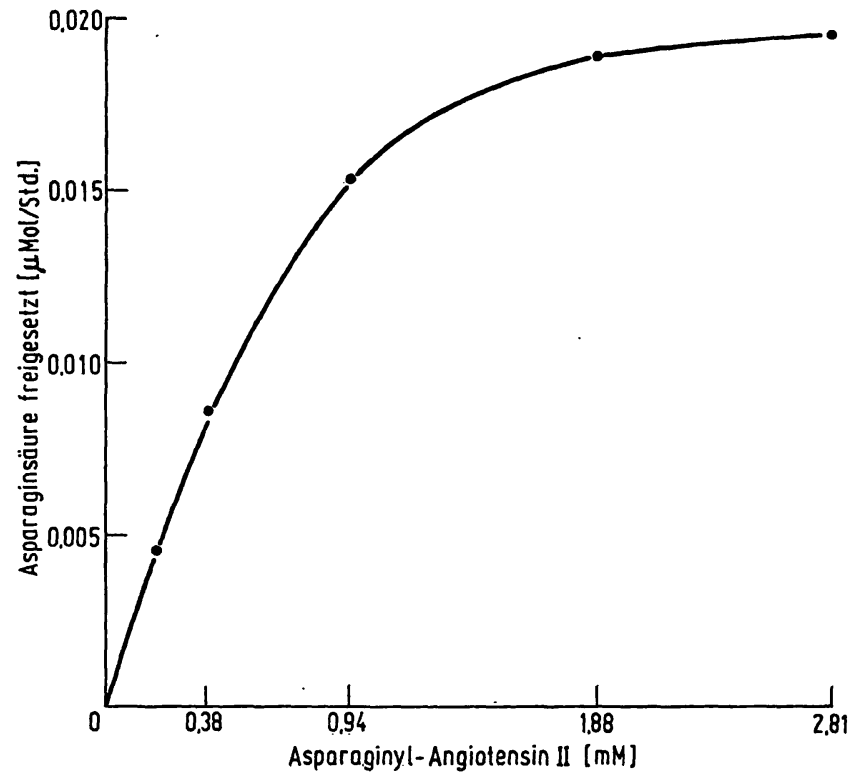

Abb. 2

Inaktivierung von Asparaginyl-Angiotensin II durch Abspaltung von

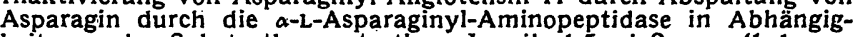
Asparagin durch die $\alpha$-L-Asparaginyl-Aminopeptidase in Abhangig-

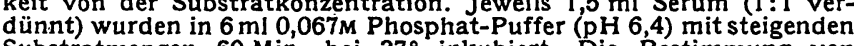
Substratmengen $60 \mathrm{Min}$. bei $37^{\circ}$ inkubiert. Die Bestimmung von Asparagin erfolgte nach Überführung in Asparaginsäure im optischen Test; Einzelheiten vgl. Methodik

\section{Präzision der Metbode}

Die Präzision wurde durch 5fach-Bestimmungen der Aktivität der $A_{1}$ AP im Serum einer Normalperson sowie im Serum eines Patienten mit Verschlußikterus ermittelt. Bei dem im Normalbereich liegenden Mittelwert von 6,22 U/1 betrug die Standardabweichung $s \pm 0,35$ und der Variationskoeffizient VK 5,6\%. Im Serum mit deutlich erhöhter Aktivität betrug bei einem Mittelwert von $32,28 \mathrm{U} / \mathrm{l}, \mathrm{s} \pm 0,85$ und VK $2,6 \%$.

\section{Normalwerte}

Die Aktivität der $A_{1}$ AP im Serum wurde bei 10 weiblichen und 15 männlichen Normalpersonen (Alter 22-48 Jahre) bestimmt. Bei den weiblichen Versuchspersonen ergaben sich Werte zwischen 4,60 und 9,70 U/1; der Mittelwert war 7,25 U/1. Bei den männlichen Personen lagen die Werte zwischen 4,17 und 10,31 U/1; der Mittelwert betrug 7,38 U/1. Der Unterschied zwischen den Normalwerten bei Frauen und Männern ist statistisch nicht signifikant. $\mathrm{Faßt}$ man beide
Gruppen zusammen, so beträgt der Mittelwert 7,33 U/1 und $s \pm 1,72$.

\section{Aktivität der Asparaginyl-Aminopeptidase bei hepatobiliären Krankbeiten}

Die Aktivität der $\mathrm{A}_{1} \mathrm{AP}$ wurde im Serum von 9 weiblichen und von 5 männlichen Patienten mit hepatobiliären Krankheiten bestimmt; bei allen Patienten bestand klinisch der Verdacht auf eine Cholostase. Die Werte für die $A_{1} A P$ lagen zwischen 11,69 und 82,87 U/1; der Mittelwert betrug 29,24 U/1. Gleichzeitig wurde bei allen 14 Patienten die Aktivität der alkalischen Phosphatase [EC 3.1.3.1] und der Alanin-Aminotransferase [EC 2.6.1.2] im Serum bestimmt. In Tabelle 1 sind die Ergebnisse dieser Untersuchungen zusammengefaßt. Um die Abhängigkeit des Anstiegs der $A_{1}$ AP-Aktivität vom Grad der Cholostase zu verdeutlichen, wurden die Patienten in drei Gruppen eingeteilt (Tab. 2):

Zur Gruppe A gehören sechs Patienten, bei denen klinisch ein Verschlußikterus oder Gallengangsstrikturen vorlagen;

Gruppe B umfaßt vier Patienten, die an einer Cholelithiasis litten;

Gruppe $C$ enthält vier Patienten, bei denen als Folge einer Lebercirrhose oder eines Pankreaskopf-Carcinoms der Verdacht auf eine intra- oder extrahepatische Cholostase bestand.

Tab. 2

Aktivitäten der $\alpha$-L-Asparaginyl-Aminopeptidase ( $A_{2} A P$ ) und $\operatorname{der}$ alkalischen Phosphatase (AP) bei 3 Patientengruppen mit Cholostase verschiedener Pathogenese

\begin{tabular}{|c|c|c|c|c|}
\hline Gruppe & Diagnose & Nr. & $\begin{array}{l}\text { Aktiv } \\
\mathrm{A}_{1} \mathrm{AP} \\
{[\mathrm{U} / 1]}\end{array}$ & $\begin{array}{c}\operatorname{der} \\
A P \\
{[U / 1]}\end{array}$ \\
\hline $\mathbf{A}$ & $\begin{array}{l}\text { Verschlußikterus, Gallengangs- } \\
\text { strikturen }\end{array}$ & $\begin{array}{r}1 \\
2 \\
4 \\
5 \\
6 \\
11 \\
\bar{x}\end{array}$ & $\begin{array}{l}82,87 \\
57,11 \\
38,49 \\
30,97 \\
26,78 \\
16,37 \\
42,10\end{array}$ & $\begin{array}{l}750 \\
340 \\
416 \\
400 \\
350 \\
212 \\
411\end{array}$ \\
\hline B & Cholelithiasis & $\begin{array}{r}3 \\
7 \\
8 \\
10 \\
\bar{x}\end{array}$ & $\begin{array}{l}39,43 \\
23,73 \\
21,45 \\
16,37 \\
25,25\end{array}$ & $\begin{array}{l}740 \\
260 \\
226 \\
432 \\
415\end{array}$ \\
\hline C & Leberparenchymerkrankungen & $\begin{array}{l}9 \\
12 \\
13 \\
14 \\
\bar{x}\end{array}$ & $\begin{array}{l}16,84 \\
13,96 \\
13,24 \\
11,69 \\
13,93\end{array}$ & $\begin{array}{l}190 \\
241 \\
154 \\
380 \\
241\end{array}$ \\
\hline
\end{tabular}


Bei allen Patienten war die Aktivität der alkalischen Phosphatase deutlich erhöht. Obgleich die hier gewählte Einteilung, die nach klinischen Gesichtspunkten erfolgte, nur orientierend sein kann, läßt sich eine Beziehung $z$ wischen der Aktivität der $A_{1} A P$ und dem $A$ usma $B$ der Cholostase erkennen. Zur Objektivierung dieser Feststellung wurde der Korrelations-Koeffizient errechnet. Es ergab sich ein Wert von $r=+0,713$ und damit eine signifikante Korrelation zwischen der Erhöhung der Aktivität der alkalischen Phosphatase und $\operatorname{der} \mathrm{A}_{1} \mathrm{AP}$ im Serum von Patienten mit Cholostase $(0,005>p$ $>0,001)$.

\section{Aktivität der Asparaginyl-Aminopeptidase im Serum wäbrend der Scburwangerscbaft}

Die Bestimmung der $A_{1}$ AP-Aktivität im Serum von 15 schwangeren Frauen im 3. Trimenon ergab Werte zwischen 2,03 und 12,65 U/1 (Tab. 3). Der Mittelwert

Tab. 3

Aktivität der $\mathrm{o}$-L-Asparaginyl-Aminopeptidase im Serum bei 15 schwangeren Frauen im 3. Trimenon

\begin{tabular}{|c|c|c|c|}
\hline \multicolumn{2}{|c|}{$\begin{array}{c}\text { Aktivität der } A_{1} A P \\
{[U / 1]}\end{array}$} & Schwangerschaft & Komplikationen \\
\hline $\begin{array}{r}2,03 \\
3,95 \\
4,03 \\
5,43 \\
6,19 \\
6,68 \\
7,14 \\
7,26 \\
7,37 \\
7,54 \\
8,21 \\
8,34 \\
11,82 \\
12,40 \\
12,65\end{array}$ & & $\begin{array}{l}\text { I. } \\
\text { IV. } \\
\text { I. } \\
\text { II. } \\
\text { I. } \\
\text { II. } \\
\text { I. } \\
\text { I. } \\
\text { III. } \\
\text { III. } \\
\text { III. } \\
\text { II. } \\
\text { III. } \\
\text { II. }\end{array}$ & $\begin{array}{l}\text { keine } \\
\text { keine } \\
\text { Kreislaufkollaps } \\
\text { Abortus imminens, Mens V } \\
\text { keine } \\
\text { keine } \\
\text { keine } \\
\text { keine } \\
\text { keine } \\
\text { keine } \\
\text { keine } \\
\text { keine } \\
\text { vaginale Blutung, Mens V } \\
\text { infektiöse Hepatitis } \\
\text { Gallenkoliken }\end{array}$ \\
\hline
\end{tabular}

lag bei $7,40 \mathrm{U} / 1$ und entsprach damit etwa dem Mittelwert im Serum der weiblichen Normalpersonen $(7,25$ U/l). Die höchsten Aktivitäten wurden bei zwei Schwangeren gemessen, von denen die eine eine infektiöse Hepatitis überstanden hatte und die andere an Gallenkoliken litt.

\section{Diskussion}

Der Nachweis einer erhöhten Aktivität der AsparaginylAminopeptidase $\left(\mathrm{A}_{1} \mathrm{AP}\right)$ bei hepatobiliären Krankheiten stimmt mit den bisherigen Angaben der Literatur (16 bis 18) überein; obwohl die einzelnen Autoren unterschiedliche Methoden benutzten, wurde als Substrat für die Inkubationen stets $\alpha$-L-Asparaginyl-Angiotensin II ( $\mathrm{Hy}$ pertensin Ciba) eingesetzt. Wie aus dem Korrelationskoeffizienten hervorgeht, konnte in der vorliegenden Arbeit eine Beziehung zwischen der erhöhten Aktivität der $\mathrm{A}_{1} \mathrm{AP}$ einerseits und der alkalischen Phosphatase im Serum andererseits nachgewiesen werden. Demnach scheint der pathogenetische Mechanismus der Aktivitätssteigerung beider Enzyme gleich oder ähnlich zu sein.

Während die alkalische Serumphosphatase hauptsächlich aus den Osteoblasten des Knochens, aus der Leber und aus dem Dünndarm stammt, ist die Aktivität der $\mathrm{A}_{1} \mathrm{AP}$ im Lebergewebe geringer als im Erythrocytenlysat, Plasma, Dünndarm und in der Niere (27). In ähnlicher Reihenfolge ist die L-Leucyl-Peptid-Hydrolase (Leucinaminpeptidase, [EC 3.4.1.1]) in den Geweben verteilt: Niere, Dünndarm, Muskel, Gehirn, Colon, Milz, Hoden, Leber, Magenschleimhaut, Pankreas und Thymus (28). Wahrscheinlich sind Darm, Niere und Erythrocyten die hauptsächlichen Bildungsorte der normal vorkommenden $A_{1}$ AP; bei Krankheiten jedoch, die mit Cholostase einhergehen, stammt möglicherweise ein Teil der erhöhten Enzymaktivität aus der Leberzelle selbst. Ein entsprechendes Verhalten wurde auch für die alkalische Phosphatase diskutiert (29).

Wie aus unseren Untersuchungen hervorgeht, scheint eine Erhöhung der $A_{1} A P$-Aktivität im Serum hauptsächlich auf eine Retention infolge der Cholostase und nicht auf Leberzellnekrosen zurückzuführen sein. Um eine Aussage über die diagnostische Brauchbarkeit der Bestimmung von $\mathrm{A}_{1} \mathrm{AP}$ machen zu können, müßte u. a. derjenige Sekretionsdruck der Galle im Ductus choledochus, der zu einem meßbaren Anstieg der $\mathrm{A}_{1} \mathrm{AP}$ Aktivität im Serum führt, ermittelt und mit demjenigen verglichen werden, bei welchem eine Erhöhung der alkalischen Serumphosphatase sowie der Leucinaminopeptidase beobachtet wird.

$\mathrm{Zu}$ erwähnen ist auch, $\mathrm{da} B$ die Leucinaminopeptidase ihrerseits ebenfalls Asparaginyl-Angiotensin II hydrolysieren kann (5) und damit zur sogenannten Angiotensinase-Aktivität beiträgt. KLAus und Mitarbeiter (8) sowie GolDACKER und OelKers (16) beschrieben einen parallelen Anstieg von Leucinaminopeptidase- und Angiotensinase-Aktivität im Serum von Leberkranken; im Serum von Schwangeren im 3. Trimenon fanden sie jedoch nur eine Erhöhung der Leucinaminopeptidase.

Die relativ niedrigen Blutdruckwerte bei Patienten mit chronischen Leberkrankheiten können wahrscheinlich nicht als Folge einer erhöhten Angiotensinase-Aktivität und damit eines beschleunigten Abbaus von Angiotensin erklärt werden; denn für die Inaktivierung des im menschlichen Organismus gebildeten Angiotensins ( $\alpha$-LAspartyl-5Isoleucin-Angiotensin II) ist nicht die $A_{1} \dot{A} P$, sondern eine andere Angiotensinase geschwindigkeitsbestimmend $(12,25,30,31,32)$. Nach Untersuchungen von LuBAsh und Mitarbeitern ist diese Angiotensinase, die als Aminopeptidase $A_{2} \quad\left(A_{2} A P, \alpha\right.$-L-AspartylAminopeptidase) bezeichnet wird, bei Leberkrankheiten nicht signifikant erhöht (33). Dagegen ist die herabgesetzte vasopressorische Reaktion auf intravenöse Infusionen von $\alpha$-L-Asparaginyl-Angiotensin II (Hypertensin Ciba) bei Patienten mit dekompensierter Lebercirrhose $(23,24)$ mit der erhöhten Aktivität der $\mathrm{A}_{1} \mathrm{AP}$ im Serum erklärbar.

Eine Erhöhung der $\mathrm{A}_{1} \mathrm{AP}$ im Serum von Schwangeren konnte in der vorliegenden Untersuchung nicht nachgewiesen werden. Dieses Ergebnis steht in scheinbarem Widerspruch zu den Befunden anderer Autoren, die eine erhöhte Angiotensinase-Aktivität im Serum während des 3. Trimenon beschrieben $(12,19-22,30)$. Die 
Methoden waren jedoch unterschiedlich: So inkubierten NAGATSU und Mitarbeiter (12) $\alpha$-L-Aspartyl- $\beta$-naphthylamid, das vom gleichen Enzym, nämlich der $\mathrm{A}_{2} \mathrm{AP}$, hydrolysiert wird wie das in vivo vorkommende AspartylAngiotensin II (12), und verfolgten die Abspaltung der endständigen Asparaginsäure mit einem kolorimetrischen Verfahren. Die übrigen Autoren $(19-22,30)$ verwendeten zwar $\alpha$-L-Asparaginyl-Angiotensin II, erfaßten jedoch bei der biologischen Messung des nicht inak- tivierten Angiotensins sowohl die Aktivität der $A_{1} A P$ als auch die der Endopeptidasen nebeneinander.

Die in der Literatur beschriebenen Ergebnisse und unsere eigenen Untersuchungen erlauben die Schlußfolgerung, daß im 3. Trimenon die Aktivität der $\alpha-\mathrm{L}-$ Aspartyl-Aminopeptidase $\left(\mathrm{A}_{2} \mathrm{AP}\right)$ und der Endopeptidasen, bei mit Cholostase einhergehenden hepatobiliären Krankheiten dagegen die Aktivität der $\alpha$-LAsparaginyl-Aminopeptidase $\left(A_{1} A P\right)$ erhöht ist.

\section{Literatur}

1. Plentl, A. A., und I. H. PAge, J. exper. Med. 79, 205 (1944). 2. RINIKER, B. und R. SchwYZER, Helv. chim. Acta 44, 658 (1961). 3. Kokubu, T., E. Ueda, S. Fujimoto, K. Hrwata, H. Sanga und Y. Yamamura, Clin. chim. Acta, Amsterdam 12, 484 (1965). 4. Yang, H. Y. T., E. G. Erdös und T. S. Chiang, Nature 218, 1224 (1968). - 5 REGoli, D., B. Riniker und H. BRuNNer, Biochem. Pharmacol. 12, 637 (1963). - 6. Khairallah, P. A., F. M. Bumpus, I. H. PAGe und R. R. Smeby, Science 140, 672 (1963). 7. Khairallah, P. A. und I. H. Page, Biochem. Med. 1, 1 (1967). 8. Klaus, D., H. Kaffarnik und H. Pfeil, Klin. Wschr. 41, 376 (1963). - 9. Hickler, R. B., D. P. LAuler und G. W. Thorn, J. clin. Invest. 42, 635 (1963). - 10. Wood, J. E., Circulation 25, 225 (1962). - 11. Worf, R. B., M. Mendlowitz, J. Pick, S. E. Gitlow und N. Naftchi, J. Laborat. clin. Med., S. Lovis 60, 150 (1962). - 12. Nagatsu, I., L. Gillespie, J. E. FolK und G. G. GleNNER, Biochem. Pharmacol. 14, 721 (1965).-13.Irzkowrtz, H.D., S. J. Dudrick, I. Dyrda und J. J. Murphy, Arch.intern.Med. 119, 241 (1967). - 14. Lubash, G. D., E. C. Hammel und R. J. Mearles, Clin. chim. Acta, Amsterdam 18, 439 (1967). - 15. Biron, P., Landesman, R. und J. C. Hunt, Nature 204, 1096 (1964). 16. Goldacker, I. U. von, und W. Oelkers, diese Z. 7, 250 (1969). - 17. Biron, P., W. P. Baldus und W. H. J. Summerskille,
Proc. Soc. exper. Biol. (N. Y.) 116, 1074 (1964). - 18. Klaus, D., H. Kaffarnik und H. Pfeil, Klin. Wschr. 41, 380 (1963). 19. Landesman, R., P. Biron, R. Castellanos, R. LaRussa und K. H. Wilson, Obstetr. Gynec. 22, 316 (1963). - 20. Berger, M. und J. Langhans, Amer. J. Obstetr. Gynec. 98, 215 (1967). 21. Talledo, O. E., Proc. Soc. exper. Biol. (N. Y.) 101, 254 (1968). 22. Morandini, G. und C. Mangioni, Acta med. scand. 185, 311 (1969). - 23. Laragh, J. H., P. C. Cannon, C. J. Bentzel, A. M. Sicinski und J. I. Meltzer, J. clin. Invest. 42, 1179 (1963). 24. Kaplan, M. und J. G. Silah, J. clin. Invest. 43, 659 (1964). 25. Oelkers, W. und I. U. von Goldacker, Klin. Wschr. 12, 649 (1967). - 26. Lineweaver, H. und D. Burk, J. Amer. chem. Soc. 56, 658 (1934). - 27. ItZkowitz, H. D. und L. MilleR, Amer. J. med. Sci. 254, 101 (1967). - 28. GreeN, M. N., K. C. Tsou, R. Bressler und A. M. Seligman, Arch. Biochem. Biophysics 57 , 458 (1955). - 29. Eisenburg, J., Fortschr. Med. 86, 903 (1968). 30. Hess, R., Biochim. biophysica Acta (Amsterdam) 99, 316 (1965). - 31. Hodge, R. L., K. K. F. NY und J. R. VANE, Nature 215, 138 (1967). - 32. Johnson, D. C. und J. W. Ryan, Biochim. biophysica Acta (Amsterdam) 160, 196 (1968). - 33. LuBASH, G. D., E. C. Hammer und R. J. Mearles, Amer. J. med. Sci. 255, 105 (1968).
Prof. Dr. H. Breuer 5300 Bonn-Venusberg Inst. f. Klin. Biochemie u. Klin. Chemie 